\title{
Symmetrization of a polygonal hollow-core vortex through beat-wave resonance
}

\author{
Hamid Ait Abderrahmane, ${ }^{1, *}$ Kamran Siddiqui, ${ }^{2}$ Georgios H. Vatistas, ${ }^{3}$ Mohamed Fayed, ${ }^{3}$ and Hoi Dick $\mathrm{Ng}^{3}$ \\ ${ }^{1}$ Department of Mechanical Engineering, McGill University, Montreal, Quebec H3A 2K6, Canada \\ ${ }^{2}$ Department of Mechanical and Materials Engineering, University of Western Ontario, London, Ontario N6A 5B9, Canada \\ ${ }^{3}$ Department of Mechanical and Industrial Engineering, Concordia University, Montreal, Quebec H3G 1M8, Canada \\ (Received 7 October 2010; revised manuscript received 24 February 2011; published 16 May 2011)
}

\begin{abstract}
We report on the symmetrization phenomenon of a hollow-core vortex in shallow liquid conditions. This phenomenon accompanies the transition of $m$ wave into $(m+1)$ wave and involves a beat-wave resonance that mediates energy transfer between the background flow and the vortex core. It is shown that this beat wave has a frequency $\frac{m}{(m-1)}$ times the frequency of the parent $m$ wave.
\end{abstract}

DOI: 10.1103/PhysRevE.83.056319

PACS number(s): 47.54.De

\section{INTRODUCTION}

Rotating shallow-water layers in enclosed cylinders are approximated as two-dimensional fluid motions and are considered as simplified laboratory models of complex geophysical and astrophysical flows [1,2]. In general, their configuration consists of inner solid-body-like rotation and outer shear flows [3]. The former fluid motion has a constant vorticity while in the latter, the vorticity and angular velocity have a negative radial gradient.

The inner flow region is susceptible to inertial instabilities which can lead to the formation of inertial waves such as Kelvin waves. However, in the outer region (shear flow), the mixing associated with some particular vorticity profiles damps flow perturbations and could produce symmetrization of the vorticity field $[4,5]$. The understanding of some aspects of the two-dimensional complex vortex dynamics, which would have been difficult to obtain with ordinary fluids, was gained through experiments on cylindrical electron plasma columns trapped in Malmberg-Penning traps, due to the analogy between the drift Poisson equations in plasma physics and Euler's equations in fluid mechanics [6,7].

The symmetrization of plasma density-the analog of the vorticity in fluid-is among those aspects investigated with trapped plasma columns. This symmetrization phenomenon, also known as downscattering, was explained as nonresonant interaction between a parent wave $\left(\omega_{m}, m\right)$ and a daughter $\left(\omega_{m-1}, m-1\right)$ wave, which leads to the formation of a beat wave $\left[\omega_{m}-\omega_{m-1}, m-(m-1)\right]$. This beat wave interacts resonantly, in a manner analogous to nonlinear Landau damping, with the background flow at the critical layer where they rotate at the same velocity $[8,9]$.

In this paper we report an experimental observation of symmetrization through beat-wave resonance of polygonal wave patterns in a hollow vortex core. The present result deepens the recent analysis of the transition between subsequent symmetrical patterns where the transition was found to occur in two steps: first, a quasiperiodic regime with two nonresonant propagating waves, followed by their synchronization and the destruction of the quasiperiodic regime at the critical conditions [10].

\footnotetext{
*haitabd@hotmail.com
}

\section{EXPERIMENTAL TECHNIQUES}

Our experiments were conducted in an apparatus consisting of a cylindrical reservoir of $284 \mathrm{~mm}$ i.d. with a flat circular disk revolving counterclockwise near the bottom of the tank $[11,12]$. When a sufficiently high swirl is imparted to a shallow layer of water by the rotating disk at the bottom of the stationary cylindrical container, a hollow-core vortex is formed. With an increase in the disk speed, the shape of the initially circular hollow core undergoes a series of transformations producing a series of $m$-wave patterns, where $m$ stands for the azimuthal wave number. Similar to Kelvin waves [13], these waves occur within the inner flow region and appear as rigid body rotating surface waves with uniform angular velocity.

To ease the extraction of the pattern contour, a blue watersoluble dye was mixed into the water prior to the experiments. A two-megapixel CCD camera (JAI CV-M2) was placed above the cylinder to image the core patterns formed on the disk. The camera was connected to a PC equipped with a digital frame grabber that acquires 8-bit images at a rate of 30 frames per second. A circular neon lamp surrounding the cylindrical tank was used to ensure uniformity of light. Circular disks of 252 and $270 \mathrm{~mm}$ diameter were used in the experiments. The experiments were conducted for the sequential transitions from $m=2$ through $m=4$. For each experimental run, 1000 images were acquired. In order to accurately detect the edges of the patterns in each image, an image processing algorithm was developed [10].

Examples of the transition between two subsequent polygonal patterns ( $m=3$ to $m=4$ and $m=2$ to $m=3$ ) are illustrated in Figs. 1 and 2, respectively. For instance, Fig. 1(a) shows the image of the stable and symmetrical parent 3 wave, obtained at the disk rotational frequency of $f_{d}=3.20 \mathrm{~Hz}$. A parent wave is defined as the intrinsic wave induced by the revolving disk at a particular disk speed. As disk speed increased, this parent wave became asymmetrical [Figs. 1(b) and 1(c)]. Once a critical disk speed was reached $\left(f_{d}=\right.$ $3.87 \mathrm{~Hz}$ ), the asymmetrical wave transformed gradually (in $\sim 20$ s or 20 laps of the wave) into a 4 -wave pattern [Figs. 1(d)1(f)]. With a further increase in disk speed, the 4 wave gradually stabilized into a symmetrical 4 wave [Figs. $1(\mathrm{~g})$ and 1(h)]. In this study, two transitions were carefully investigated. Because of the space limitation, emphasis is placed on the 


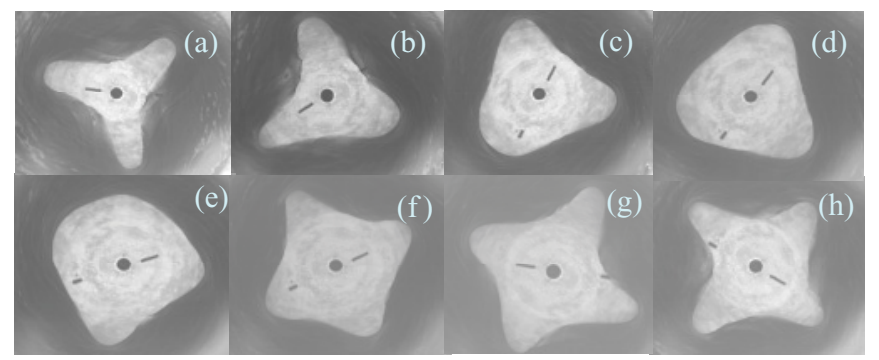

FIG. 1. Transition from 3-wave to 4-wave pattern. (a)-(c) As the disk speed increases, the area of the pattern increases. (b) and (c) The initial symmetrical 3-wave pattern becomes asymmetrical because of the faster beat wave rotating around it. (d)-(f) The critical value of the disk speed is reached and the transformation into quasisquare is in progress. (g) State before the completion of the transition. (h) Transition is completed and the pattern set in a symmetrical square shape. Mean water height above the rotating disk: $h_{0}=40 \mathrm{~mm}$; disk diam: $d=270 \mathrm{~mm}$.

transition from 3 wave to 4 wave. The transition of 2 wave into 3 wave as seen in Fig. 2 is briefly discussed in this paper for completeness; Nevertheless, it is found that in both cases, the transition follows the same mechanism. It is worth noting that the vortex-core changeovers at higher modes were difficult or even impossible to investigate because the range of the disk speed where these higher modes exist is very narrow and hence extremely challenging to capture experimentally.

\section{DATA ANALYSIS}

The examination of the symmetrization process was based on the analysis of the time series of the radial displacement, $r(t)$, at a given point on the pattern's edge, measured from the disk center. This analysis was conducted in two frames of reference; one was the laboratory frame of reference while the second one was the relative frame of reference (moving with the parent wave pattern). In the laboratory frame of reference, the time series $r(t)$ was decomposed into its constituent parts, using the empirical mode decomposition (EMD) technique [14]. The time series in the relative frame of reference were analyzed through spectral analysis.

In the laboratory frame of reference, only the first and second fundamental intrinsic mode functions (IMFs) were relevant. The amplitudes of the other IMFs were very small and thus ignored. The normalized amplitudes of the IMFs are depicted in Figs. 3 and 4. Figures 3(a) and 3(b) show the time series and the IMFs that correspond to the patterns seen in Fig. 1(a). The first intrinsic mode function corresponds to the rotation of the 3 wave about the center of the disk while the second one corresponds to a secondary wave that modulates

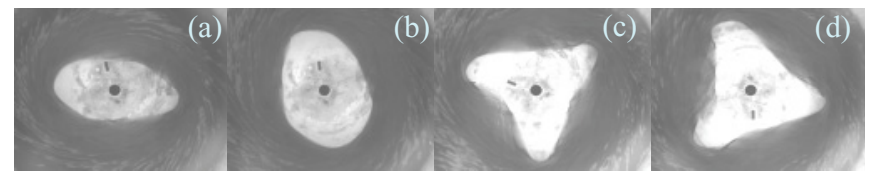

FIG. 2. Transition from 2-wave to 3-wave pattern. (a) The initial 2-wave pattern. (b) and (c) Transition from 2 wave into 3 wave. (d) Transition is completed. Mean water height above the rotatingdisk: $h_{0}=30 \mathrm{~mm}$; disk diam: $d=252 \mathrm{~mm}$. the 3 wave. The period of the second IMF $\left(T_{2}=0.6 \mathrm{~s}\right)$ is twice the period of that of the first IMF $\left(T_{1}=0.3 \mathrm{~s}\right)$. At the initial stage, the amplitude of the second IMF is small relative to the first IMF [Fig. 3(b)], that is, its effect is not perceptible on the pattern contour; the pattern is symmetrical [Fig. 1(a)]. While increasing the disk speed, the amplitude of the modulating wave [second IMF in Fig. 3(d)] increases and the symmetry of the 3-wave pattern breaks [Figs. 1(b)-1(d)]. Similarly, Fig. 4 shows a time series and its first intrinsic mode decomposition for the 2 wave as shown in Fig. 2(a). In this case, the time series displays only a single prominent IMF; the amplitudes of the others IMFs are very small. As it will be shown later the modulating wave (which should be a second IMF) coincides with the parent wave; their frequencies are locked in a ratio equal to 2 .

The power spectrum of the raw time series, $r(t)$ in Fig. 3(c) (in the laboratory frame of reference), is shown in Fig. 5(a). Two frequencies, $f_{3}=3.34 \mathrm{~Hz}$ and $f_{4}=4.98 \mathrm{~Hz}$, are prominent; from the image analysis before and after the transition one can easily relate $f_{3}$ and $f_{4}$ to the parent 3 waves
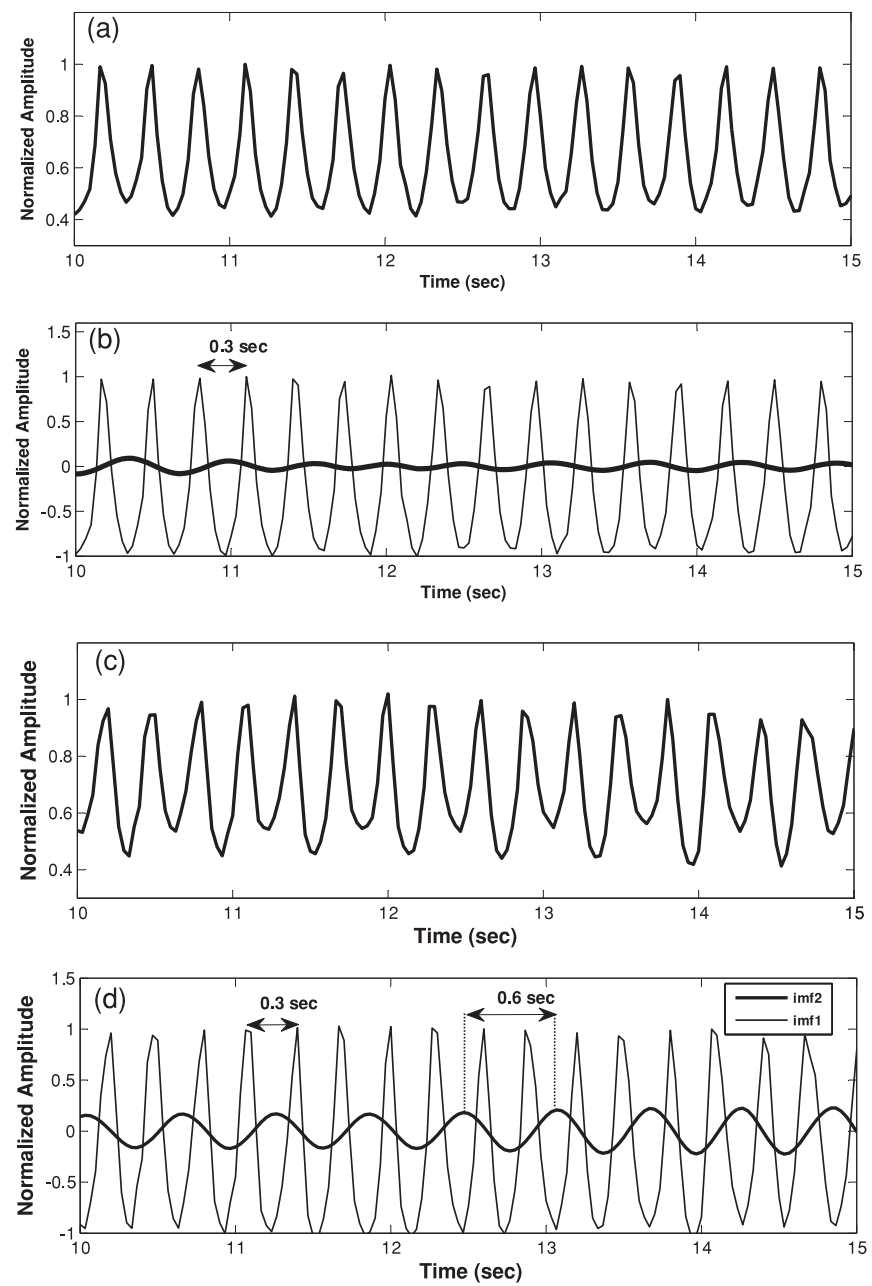

FIG. 3. Intrinsic mode functions obtained from the decomposition of the time series in the laboratory frame of reference during the transition from 3 wave into 4 wave [the results correspond to the wave pattern shown in Figs. 1(a) and 1(b)]. (a) and (c) Raw time series. (b) and (d) The first intrinsic mode decomposition of the raw time series in (a) and (c), respectively. 

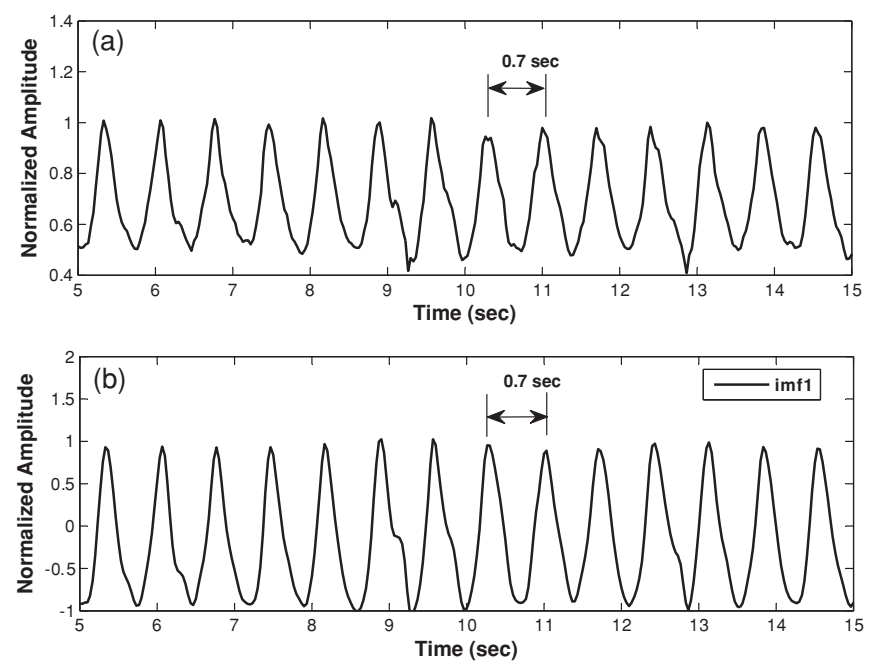

FIG. 4. Intrinsic mode functions obtained from the decomposition of the time series in the laboratory frame of reference during the transition from 2 wave into 3 wave [the results correspond to the wave pattern shown in Fig. 2(a)]. (a) Raw time series. (b) The first intrinsic mode decomposition of the raw time series.

and background (daughter) 4 waves, respectively [10]. The parent wave is the wave which is visible (3 wave in the present case), while the daughter or background wave is not visible. The effect of this daughter wave is perceptible through its interaction with the parent wave; this interaction produces a beat wave. The spectrum in Fig. 5(a) provides clear evidence of this beat-wave phenomenon through the manifestation of a high amplitude signal at the frequency which is the exact difference of the two fundamental (parent and daughter) frequencies. That is, $f_{b}=f_{4}-f_{3}=1.64 \mathrm{~Hz}$ and its wave number is $(m+1)-m=1$. The presence of this beat wave is also evident through the empirical mode decomposition of the raw time series $r(t)$ in Fig. 3. The first IMF with period $T_{1}=0.3 \mathrm{~s} \approx \frac{1}{f_{3}} \approx \frac{1}{3.34 \mathrm{~Hz}}$ is the parent mode, while the second IMF with period $T_{2}=0.6 \mathrm{~s}=\frac{1}{f_{b}}=\frac{1}{1.64 \mathrm{~Hz}}$ is the beat wave that travels faster around a pattern which rotates at a frequency $f_{p}=\frac{f_{3}}{3}$. This beat wave causes the dissymmetry of the parent pattern.

The power spectrum of the time series in Fig. 4(a) (in the laboratory frame of reference) for the 2 wave is shown in Fig. 5(b). Similar to Fig. 5(a), this power spectrum exhibits two prominent frequencies, $f_{2}=1.406 \mathrm{~Hz}$ and $f_{3}=2.871 \mathrm{~Hz}$, and the beat frequency, i.e., $f_{b} \approx f_{3}-f_{2}=1.465 \mathrm{~Hz}$. The values of $f_{b}$ and $f_{2}$ are very close to each other. Considering the uncertainties in the frequency resolution of the power spectrum $(0.0586 \mathrm{~Hz})$, it is reasonable to assume that the frequencies of the parent and beat waves coincide. This explains why the second IMF (beat wave) of period $T \approx 0.7 \mathrm{~s}$ is not observed in Fig. 4(b).

The analysis of the time series in the frame of reference moving with the parent 3 wave, rotating at a frequency $f_{p}=\frac{f_{m}}{m}$ is conducted using spectral analysis. Figure 6 shows the power spectrum at different disk speeds that cover the range from 3-wave stable state through transition to 4-wave stable state. Note that, as the frame of reference is rotating at the frequency of the pattern, the first intrinsic mode (which is dominant in
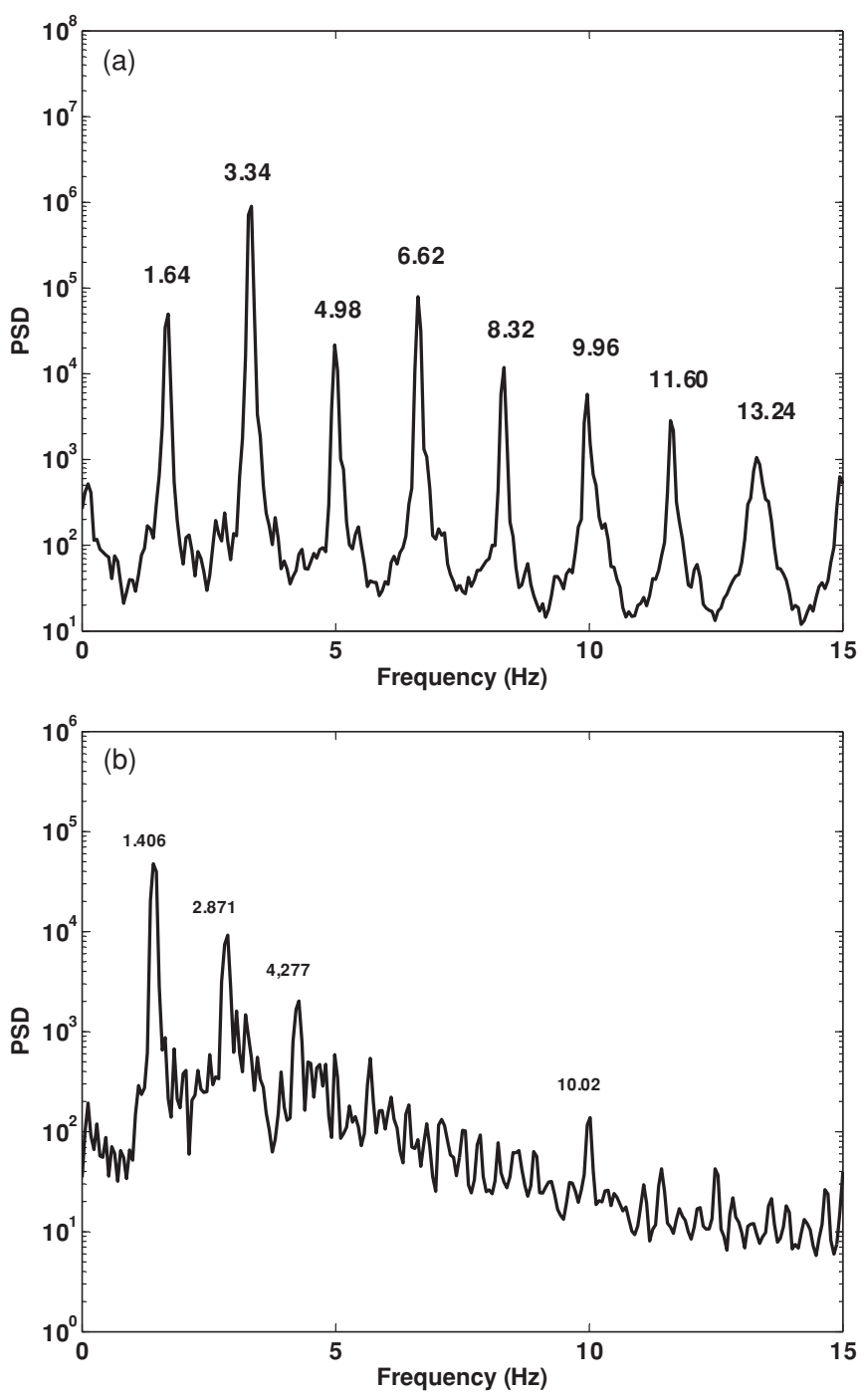

FIG. 5. Power spectrum of the time series of the radial displacement at a given point on the wave-pattern edge. The time series was measured in the laboratory frame of reference. (a) Power spectrum corresponding to the time series decomposed in Fig. 3(a) and the rotating pattern in Fig. 1(a); disk speed: $f_{\mathrm{d}}=3.64 \mathrm{~Hz}$. (b) Power spectrum corresponding to the time series decomposed in Fig. 4(a) and the rotation pattern in Fig. 2(a); disk speed: $f_{\mathrm{d}}=2.2 \mathrm{~Hz}$. The frequency resolution is $0.0586 \mathrm{~Hz}$ and the precision of the peaks is $\sim \pm 0.03$.

the laboratory frame of reference) will not manifest in these spectra. At the disk speed of $3.2 \mathrm{~Hz}$, which corresponds to the 3-wave stable state, no dominant frequency appeared in the spectrum. As mentioned earlier, the first intrinsic mode is not present and the second intrinsic mode has very low amplitude. Due to the absence of the beat wave, the pattern is symmetric [see Fig. 1(a)]. As the disk speed increased, a dominant wave appeared in the spectra at a frequency of $0.58 \mathrm{~Hz}$. A beat wave in the frame of reference moving with the pattern would appear at a frequency that is the difference between the beatwave frequency in the laboratory frame of reference and the frequency of the rotating frame of reference, i.e., $f_{b}-f_{p}=$ $f_{b}-\frac{f_{m}}{m}$. Considering the spectral resolution, the dominant frequency at $0.58 \mathrm{~Hz}$ is indeed the frequency of the beat wave 

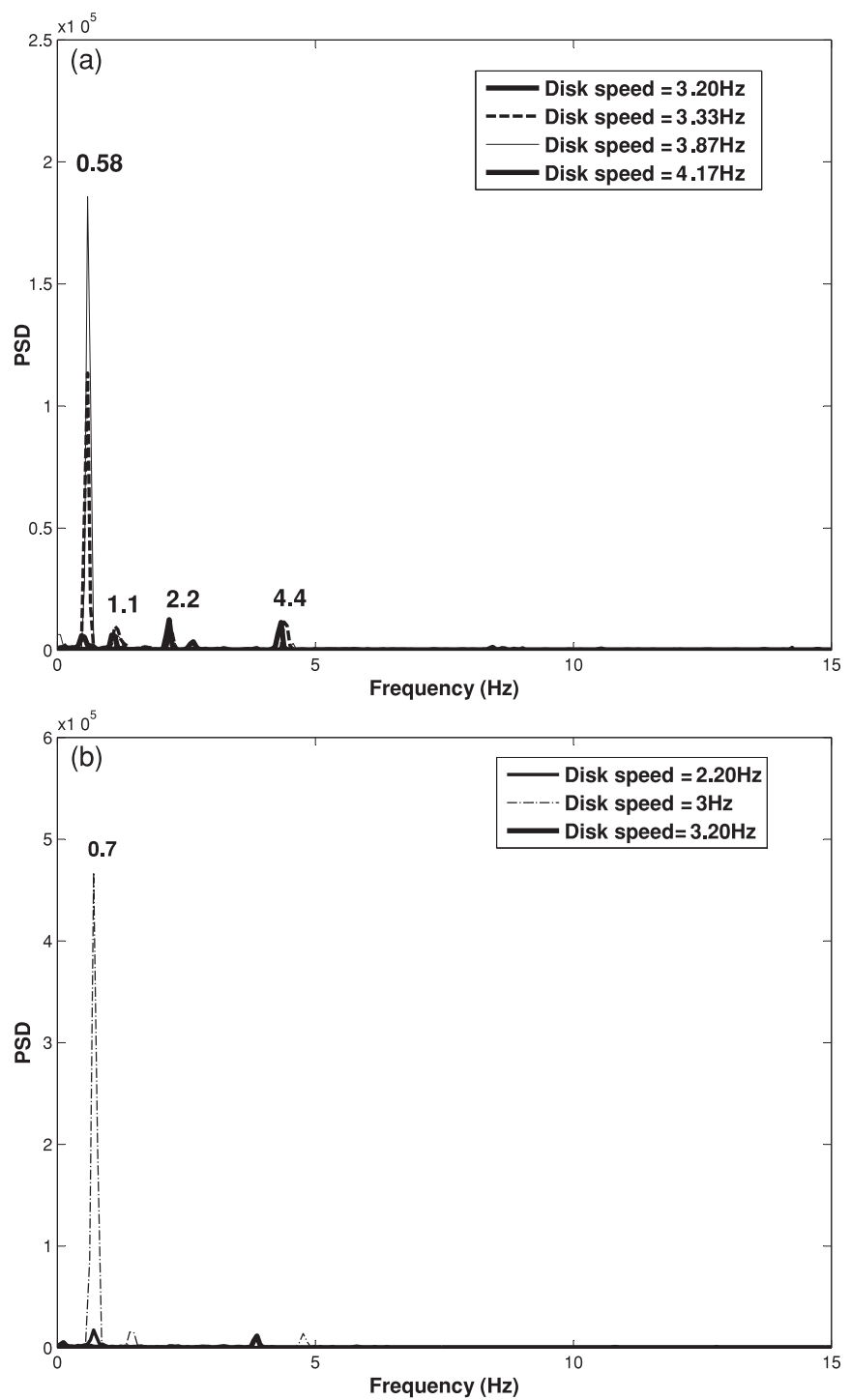

FIG. 6. Power spectra of the time series of the radial displacement at a given point on the wave-pattern edge. The time series was measured in the relative frame of reference traveling with the parent $m$ wave. The power spectra show that the amplitude of the main frequency increases as it approaches the critical speed at which the transformation from $m$ wave into $(m+1)$ wave occurs. After this transition the amplitude of the main frequency decreases until it becomes almost negligible. The frequency resolution is $0.0586 \mathrm{~Hz}$ and the precision of the peaks is $\sim \pm 0.0586 \mathrm{~Hz}$. (a) Transition from 3 wave into 4 wave. (b) Transition from 2 wave into 3 wave.

that travels around the parent 3 wave (second IMF) and causes the pattern's dissymmetry leading to the transition from 3 wave to 4 wave [see Figs. 1(b)-1(e)]. The frequency of this beat wave can be related to the parent wave frequency as $f_{b}=\frac{3}{2} f_{p}$. Figure 6(a) shows that as the disk speed gets closer to its critical value $\left(f_{d}=3.87 \mathrm{~Hz}\right)$ at which a transformation from 3 wave to 4 wave occurs, the amplitude of the beat wave reaches maximum. After the transformation, the amplitude of the beat wave decreased until it became almost negligible when the 4 wave set in [see spectrum in Fig. 6(a) at $4.17 \mathrm{~Hz}$ ].

A similar mechanism was observed during the transition from 2 wave into 3 wave. The modulating wave traveling

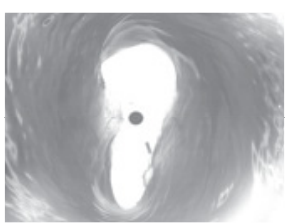

Initial

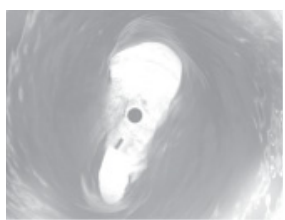

Half lap

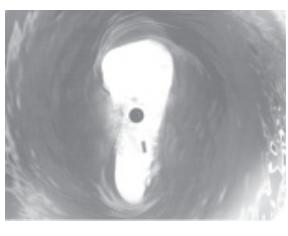

Complete lap
FIG. 7. A beat wave that travels two times faster than the parent 2 wave. The beat wave completes two laps while the 2-wave pattern completes one rotation. Experimental conditions: mean water height above the rotating disc: $h_{0}=30 \mathrm{~mm}$; disk diam: $d=252 \mathrm{~mm}$.

around the 2-wave parent wave was also analyzed in the rotating frame of reference corotating with the 2 wave. The power spectrum in this relative frame of reference [Fig. 6(b)] indicates that the frequency of the modulating wave is $f_{s}=$ $0.7 \mathrm{~Hz}$. This frequency corresponds to $f_{b}-f_{p}=f_{b}-\frac{f_{m}}{m}$, where $m=2$, which is indeed a beat wave. The frequency of this beat wave can also be related to the parent wave frequency as $f_{b} \approx 2 f_{p}$. This indicates that the beat wave is two times faster than the parent wave; see Fig. 7, which shows that a beat wave completes two laps while the 2 -wave pattern completes one rotation. Similarly, the power spectrum in Fig. 6(b) shows that the amplitude of the beat wave reached its maximum value at the critical disk speed $\left(f_{d}=3 \mathrm{~Hz}\right)$ and decreased substantially in amplitude after the transition, i.e., when the 3-wave pattern set in.

From the above observations, one can expect that the beat wave plays a key role in the transition process from $m$ wave into $(m+1)$ wave. In fact, as the disk speed comes closer to its critical value, i.e., the value at which the transition occurs, the amplitude of the beat wave increases substantially; see, for instance Fig. 3. At the critical speed the amplitude increases continuously until it reaches its maximum at the end of the transition process, i.e., when the daughter wave takes over
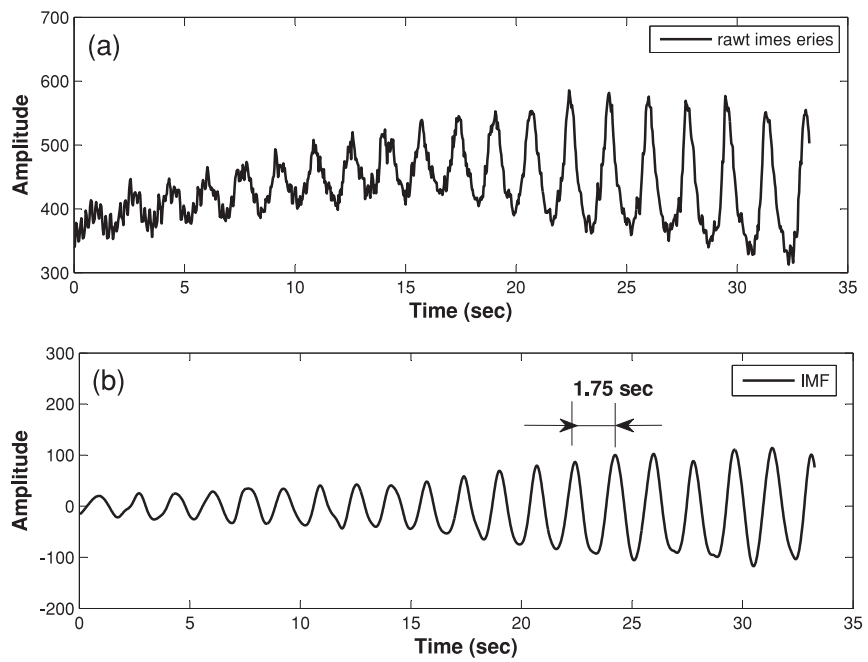

FIG. 8. Time series of the radial displacement at a given point on the wave-pattern edge, measured in the relative frame of reference rotating with the parent 3 wave. The amplitude of the modulating beat wave increases continuously, which mimics a resonance phenomenon. (a) Raw time series. (b) First empirical mode of the time series. 

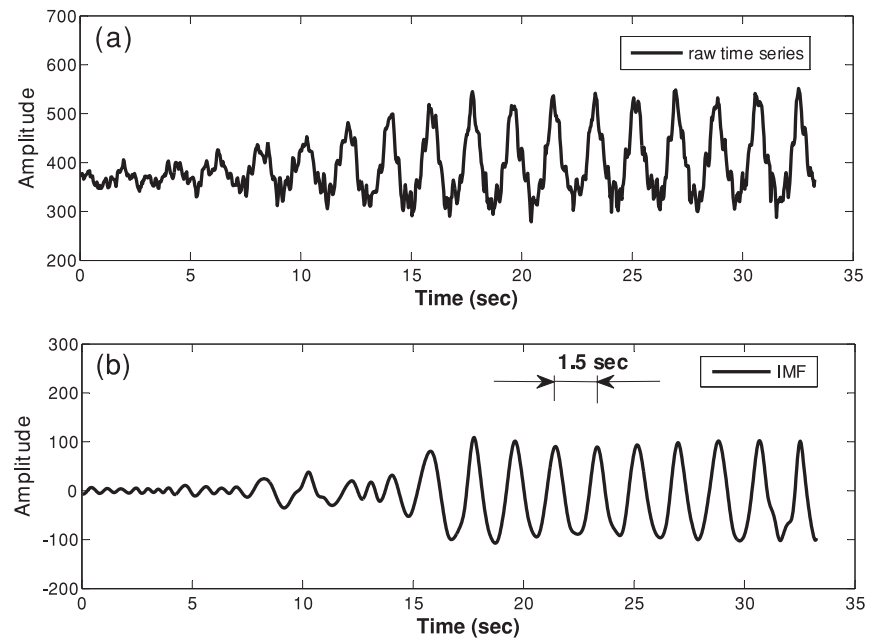

FIG. 9. Time series of the radial displacement at a given point on the wave-pattern edge, measured in the relative frame of reference rotating with the parent 2 wave. The amplitude of the modulating beat wave increases continuously, which mimics a resonance phenomenon. (a) raw time series. (b) First empirical mode of the time series.

and replaces the parent wave and becomes the visible mode. The behavior is clearly evident in Figs. 8 and 9 that show the time history of the amplitude variation of the beat wave at the critical speeds for the transition from 3 wave into 4 wave and from 2 wave into 3 wave, respectively.

This continuous increase of beat-wave amplitude as depicted in Figs. 8 and 9, and their corresponding power spectrum in Fig. 6 mimic a resonance phenomenon. The parent and daughter waves are nonresonant, i.e., $\left(f_{3} \neq f_{4}\right)$ and $\left(f_{2} \neq f_{3}\right)$. The beat wave in each of the two transitions discussed previously is not resonant with either the parent wave $\left(f_{b} \neq f_{m}\right)$ or the daughter wave $\left(f_{b} \neq f_{(m+1)}\right)$. Therefore the transition between the patterns should follow a path other than the direct resonance between the interacting waves. Keeping in mind the prominent role of resonant wave-fluid interaction at "critical layer" [15] on the evolution of a surface wave on a circular vortex [8], we conjecture that a similar process, i.e., the resonance of the beat wave with the background fluid, is the mechanism of the symmetrization of the hollow-core vortex. This resonance should occur at the "critical layer" where the beat wave and the background fluid have the same angular velocity, i.e., $f_{b}=\Omega\left(r_{c}\right)$. In this condition, the beat wave would extract energy from the background fluid surrounding the pattern and would reach an amplitude that would trigger the transition from lower to higher mode. Once the transition is completed, this high energetic fluid associated with the beat wave becomes a part of the higher-mode vortex core. Further research is needed to verify this conjecture through the investigation of the vorticity profile in the flow field using advanced measurement techniques to further confirm that the beat-wave-fluid interaction is the best explanation for the exponential growth of the beat-wave amplitude as observed in Figs. 8 and 9.

\section{CONCLUDING REMARKS}

The role of the viscosity during this symmetrization process of the hollow-core vortex was studied by increasing the viscosity of the water up to 16 times by adding glycerol. The power spectra during the symmetrization from this case resemble closely those obtained with water [10]. The process of pattern formation and their transition also involved parent, daughter, and beating waves similar to the water case. This corroborates a comment in Jansson et al. [16] on the negligible influence of the viscosity on the pattern formation. However, we observe that the viscosity modifies the value of the disk speed at which a given $m$ mode sets in. For instance, when the viscosity of the fluid is fifteen times the viscosity of the water, the disk range where the 3 mode exists is reduced by $\sim 40 \%$. In summary, one can infer that the symmetrization, described in this paper, is apparently an inviscid phenomenon similar to the "inviscid damping" [17], which reinforces our conjecture that the underlying mechanism is beat-wave-fluid interactionwhich is also known as beat-wave resonant, downscattering, nonresonant mode-mode coupling, and nonlinear Landau damping in plasma physics.
[1] A. M. Fridman, A. G. Morozov, M. V. Nezlin, and E. N. Snezhkin, Phys. Lett. A 109, 228 (1985).

[2] E. V. Guslyakova, Fluid Dyn. 28, 638 (1993).

[3] J. M. Lopez, F. Marques, A. H. Hirsa, and R. Miraghaie, J. Fluid Mech. 502, 99 (2004).

[4] A. David, D. A. Bachman, and R. W. Gould, IEEE Trans. Plasma Sci. 24, 14 (1996).

[5] N. S. Pillai and R. W. Gould, Phys. Rev. Lett. 73, 2849 (1994).

[6] K. S. Fine, A. C. Cass, W. G. Flynn, and C. F. Driscoll, Phys. Rev. Lett. 75, 3277 (1995).

[7] K. S. Fine, C. F. Driscoll, J. H. Malmberg, and T. B. Mitchell, Phys Rev Lett. 67, 588 (1991).

[8] T. B. Mitchell and C. F. Driscoll, Phys. Rev. Lett. 73, 2196 (1994).

[9] N. Mattor, B. T. Chang, and T. B. Mitchell, Phys. Rev. Lett. 96, 045003 (2006)
[10] H. Ait Abderrahamne, K. Siddiqui, and G. H. Vatistas, Phys. Rev. E 80, 066305 (2009).

[11] G. H. Vatistas, J. Fluid Mech. 217, 241 (1990)

[12] G. H Vatistas, H Ait Abderrahmane, and K Siddiqui, Phys Rev. Lett. 100, 174503 (2008).

[13] W. Thomson (Lord Kelvin), Phil. Mag. 10, 155 (1880).

[14] N. E. Huang, Z. Shen, S. R. Long, M. C. Wu, H. H. Shih, Q. Zheng, N. C. Yen, C. C. Tung, and H. H. Liu, Proc. Roy. Soc. Lond. A 454, 903 (1998).

[15] R. J. Briggs, J. D Daugherty, and R. H. Levy, Phys. Fluids 13, 421 (1970).

[16] T. R. N. Jansson, M. P. Haspang, K. H. Jensen, P. Hersen, and T. Bohr, Phys. Rev. Lett. 96, 174502 (2006).

[17] D. A. Schecter, D. H. E. Dubin, A. C. Cass, C. F. Driscoll, I. M. Lansky, and T. M. O’Neil, Phys. Fluids 12, 2397 (2000). 